International Journal of Pure and Applied Mathematics

Volume 116 No. 1 2017, 105-113

ISSN: 1311-8080 (printed version); ISSN: 1314-3395 (on-line version)

url: http://www.ijpam.eu

doi: $10.12732 /$ ijpam.v116i1.10

ijpam.eu

\title{
CONNECTED DOMINATION PATH DECOMPOSITION OF TRIANGULAR SNAKE GRAPH
}

\author{
E. Ebin Raja Merly ${ }^{1}$, D. Jeya Jothi ${ }^{2} \S$ \\ ${ }^{1,2}$ Nesamony Memorial Christian College \\ Marthandam, Tamil Nadu, 629165, INDIA
}

\begin{abstract}
In this paper, we unite connected domination and decomposition to introduce a new concept called connected domination decomposition (CDD). We also establish connected domination path decomposition of a graph G. In particular, we investigate connected domination path decomposition of triangular snake $T_{m}$.
\end{abstract}

AMS Subject Classification: $97 \mathrm{~K} 30$

Key Words: decomposition, domination, connected domination, connected domination decomposition, connected domination path decomposition

\section{Introduction}

All basic terminologies from graph theory are used in this paper in the sense of Frank Harary [4]. By a graph considered here are simple undirected graph without loops or multiple edges. As usual $p, q, m$ and $s$ denote the vertices, edges, blocks and cut vertices of a graph $G$ respectively.

The theory of domination is one of the fastest growing area in graph theory. Which has been investigated by S.T. Hedetniemi, C.J. Cockayne et al.

A set $D \subseteq V$ of vertices in a graph $G$ is a dominating set if every vertex $v$ in $V-D$ is adjacent to a vertex in $D$. The minimum cardinality of a dominating set of $G$ is called the domination number of $G$ and is denoted by $\gamma(G)$. C.J. Cockayne and S.T. Hedetniemi was introduced the notation $\gamma(G)$ in 1977.

Received: $\quad$ March 15, 2017

Revised: July 14, 2017

Published: August 29, 2017

${ }^{\S}$ Correspondence author (c) 2017 Academic Publications, Ltd. url: www.acadpubl.eu 
A dominating set $D$ of a graph $G$ is a connected dominating set if the induced subgraph $\langle D\rangle$ is connected. The number of vertices in a minimum connected dominating set is defined as the Connected Domination number of a graph $G$ and it is denoted by $\gamma_{c}(G)$. This concept was introduced by Sampathkumar in [8].

Another important area of a graph theory is decomposition of graphs.

Several authors studied various types of decompositions by imposing conditions on $G_{i}$ in the decomposition.

Let $G=(V, E)$ be a simple connected graph with $p$ vertices and $q$ edges. If $G_{1}, G_{2}, \ldots, G_{n}$ are connected edge disjoint subgraphs of $G$ with $E(G)=$ $E\left(G_{1}\right) \cup E\left(G_{2}\right) \cup \ldots \cup E\left(G_{n}\right)$, then $\left(G_{1}, G_{2}, \ldots, G_{n}\right)$ is said to be a Decomposition of $G$.

We introduce a new concept connected domination decomposition of a graph which is motivated by the concepts of Ascending Subgraph Decomposition [1], Continuous Monotonic Decomposition [3] and Ascending Domination Decomposition [6].

A vertex $v$ is called a cut vertex if removing it from $G$ increases the number of components of $G$. Here $S$ is the set of cut vertices and $s(G)=|S|$. A connected graph that has no cut vertices is called a block $(B)$ and $m(G)=|B|$. For a connected graph $G$ with blocks $\left\{B_{i}\right\}$ and cut vertices $\left\{c_{j}\right\}$ the block cut point graph of $G$ denoted by $b c(G)$ is defined as the graph having vertex set $\left\{B_{i}\right\} \cup\left\{c_{j}\right\}$ with two vertices adjacent if one corresponds to a block $B_{i}$ and the other to a cut vertex $c_{j}$ and $c_{j}$ is in $B_{i}$. The neighborhood of a vertex $u$ is the set $N(u)$ consisting of all vertices $v$ which are adjacent with $u$.

An Umbrella graph $U(m, n)$ to be a graph obtained from a fan by joining a path of length $\mathrm{m}, P_{m}$ to a middle vertex of a path $P_{n}$. A Web graph $\left(W_{n}\right)$ is a prism graph $Y_{m, n}$ with the edges of the outer cycle removed. The Helm graph $H_{n}$ is obtained from a wheel by attaching a pendant vertex at each vertex of the $n$-cycle.

\section{Connected Domination Decomposition (CDD)}

Definition 2.1. A decomposition $\left(G_{1}, G_{2}, \ldots, G_{n}\right)$ of $G$ is said to be CDD if:

(i) $E(G)=E\left(G_{1}\right) \cup E\left(G_{2}\right) \cup \ldots \cup E\left(G_{n}\right)$

(ii) Each $G_{i}$ is connected 


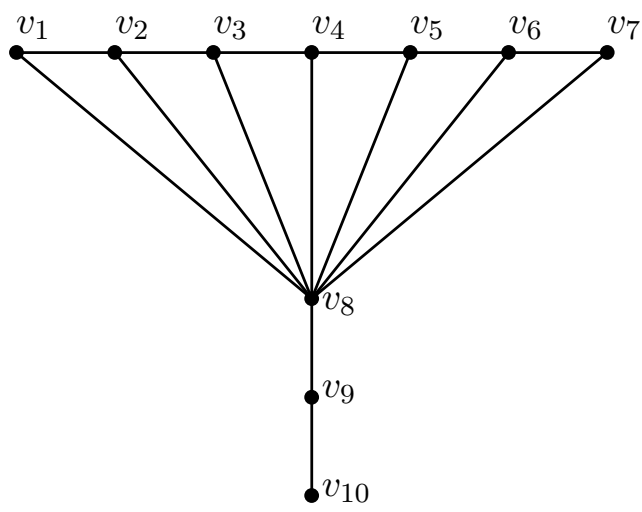

$G_{1}$

$G_{2}$

$G_{3}$
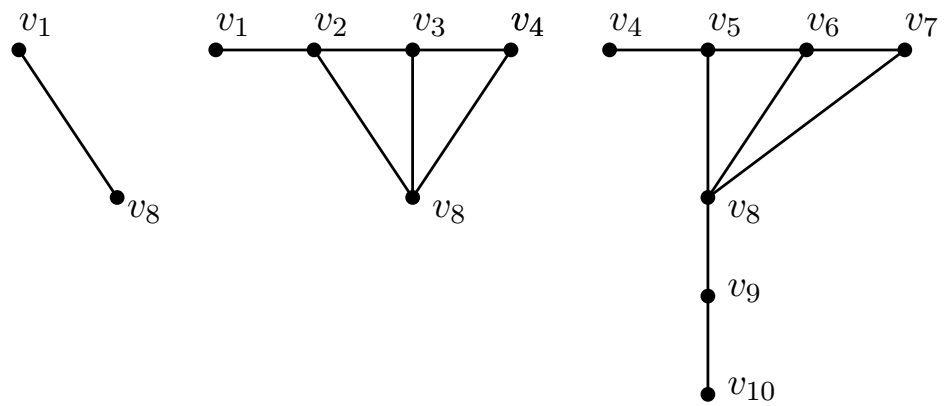

Figure 1

(iii) $\gamma_{c}\left(G_{i}\right)=i, 1 \leq i \leq n$.

Example 2.2. The umbrella graph $U(7,3)$ admits $\operatorname{CDD}\left(G_{1}, G_{2}, G_{3}\right)$ is illustrated in Figure 1.

\section{Connected Domination Path Decomposition (CDPD)}

Definition 3.1. A decomposition $\left(G_{1}, G_{2}, \ldots, G_{n}\right)$ of $G$ is said to be CDPD if:

(i) $G$ admits CDD, 

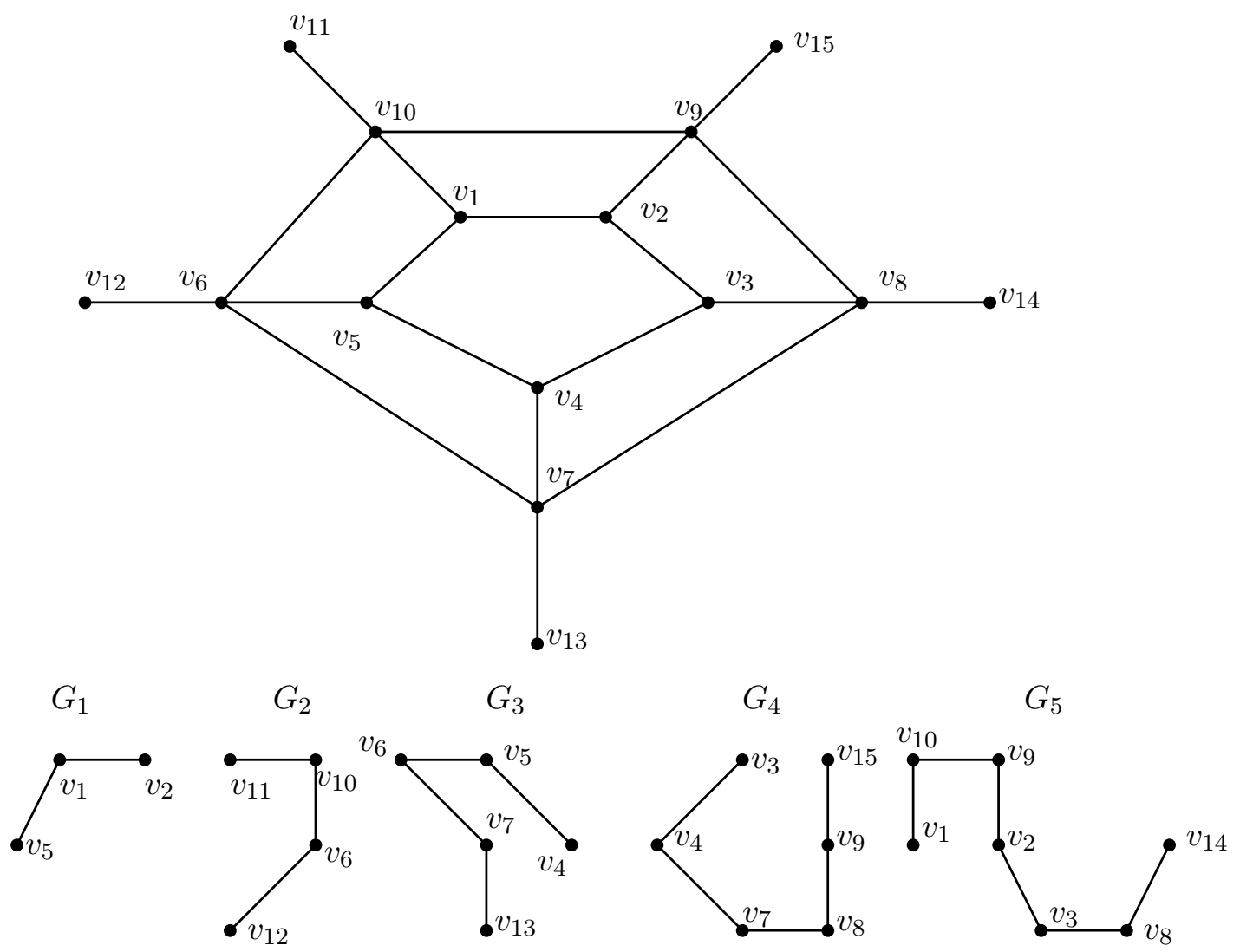

Figure 2

(ii) $q\left(G_{1}\right)=1$ or 2 ,

(iii) $q\left(G_{i}\right)=i+1, i=2,3, \ldots, n$,

(iv) each $G_{i}(1 \leq i \leq n)$ is a path.

Example 3.2. The Web graph $W_{5}$ admits $\operatorname{CDPD}\left(G_{1}, G_{2}, G_{3}, G_{4}, G_{5}\right)$ is illustrated in Figure 2.

Theorem 3.3. If a graph $G$ admits $C D P D\left(G_{1}, G_{2}, \ldots, G_{n}\right)$, then

$$
q(G)= \begin{cases}\frac{n(n+3)}{2}-1, & \text { if } q\left(G_{1}\right)=1, \forall n \in N \\ \frac{n(n+3)}{2}, & \text { if } q\left(G_{1}\right)=2, \forall n \in N\end{cases}
$$


Proof. Assume that $G$ admits CDPD. Therefore, $q\left(G_{1}\right)=1$ or 2 and $q\left(G_{i}\right)=i+1, i=2,3, \ldots, n$.

Case 1. $q\left(G_{1}\right)=1$.

$$
\begin{aligned}
q(G) & =q\left(G_{1}\right)+q\left(G_{2}\right)+\ldots+q\left(G_{n}\right) \\
& =1+[3+4+\ldots+(n+1)] .
\end{aligned}
$$

Therefore, $q(G)=\frac{n(n+3)}{2}-1$.

Case 2. $q\left(G_{1}\right)=2$.

$$
\begin{aligned}
q(G) & =q\left(G_{1}\right)+q\left(G_{2}\right)+\ldots+q\left(G_{n}\right) \\
& =2+[3+\ldots+(n+1)] .
\end{aligned}
$$

Therefore, $q(G)=\frac{n(n+3)}{2}$.

Remark 3.4. In the above theorem: For case $1, G_{1}=P_{2}, G_{2}=P_{4}, G_{3}$ $=P_{5}, \ldots, G_{n}=P_{n+2}(n \geq 2)$ are the decompositions of $G$.

In case $2, G_{1}=P_{3}, G_{2}=P_{4}, G_{3}=P_{5}, \ldots, G_{n}=P_{n+2}$ are the decompositions $G$.

Remark 3.5. The converse of the above theorem need not be true. For the Helm graph $\left(H_{9}\right)$,

$q\left(H_{9}\right)=27.27$ is the sum of $2,3,4,5,6$ and 7 . But $H_{9}$ does not admit CDPD because each $G_{i}, i=1,2, \ldots, 6$ is not a path.

\section{Connected Domination Path Decomposition of Triangular Snake Graph $\left(T_{m}\right)$}

A triangular snake is a connected graph all of whose blocks are triangles. A triangular snake is a triangular cactus whose block cut point graph is a path. Equivalently it is obtained from a path $P=v_{1}, v_{2}, \ldots, v_{m+1}$ by joining $v_{i}$ and $v_{i+1}$ to a new vertex $u_{i}, i=1,2, \ldots, m$. A triangular snake has $2 m+1$ vertices and $3 m$ edges, where $m$ is the number of blocks in the triangular snake. We denote this triangular snake by $T_{m}$. Here $v_{1}, v_{2}, \ldots, v_{m+1}$ is a set of base vertices of $T_{m}$. Since $q\left(T_{m}\right)=3 m, q\left(G_{1}\right)$ must be 2 .

Theorem 4.1. If a triangular snake $T_{m}$ admits $C D P D\left(P_{3}, P_{4}, P_{5}, \ldots\right.$, $\left.P_{3 i+2}\right)$, then $q\left(T_{m}\right)=\frac{(9 i(i+1))}{2}, i \in N$. 


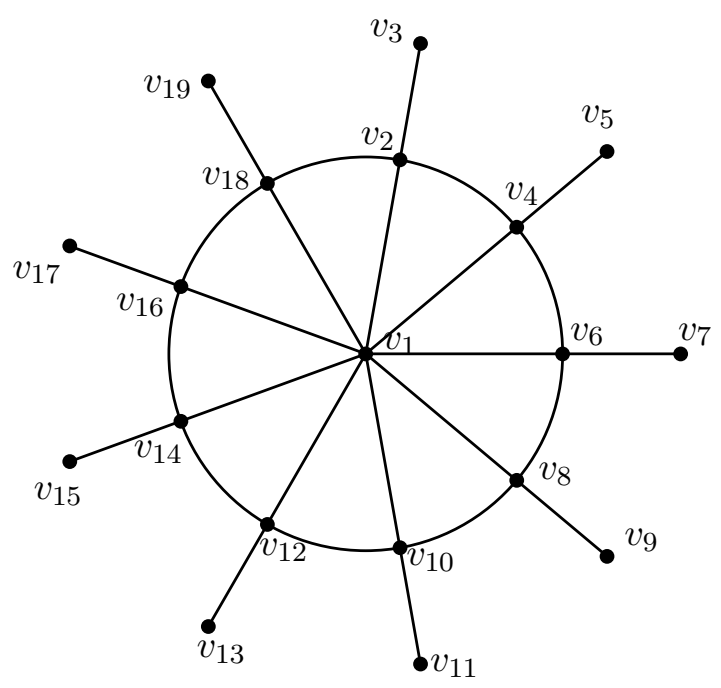

Figure 3

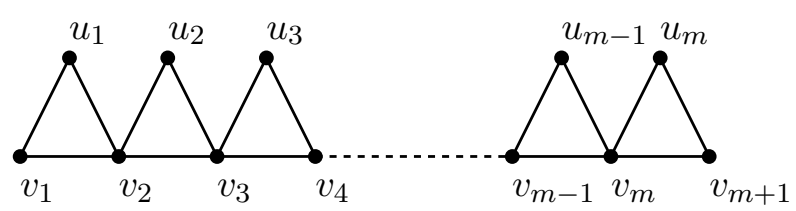

Figure 4

Proof. Assume that $T_{m}$ admits $\operatorname{CDPD}\left(P_{3}, P_{4}, P_{5} \ldots, P_{3 i+2}\right)$. Here $G_{1}=$ $P_{3}, G_{2}=P_{4}, G_{3}=P_{5}, \ldots, G_{3 i}=P_{3 i+2}$. By Theorem $(3.3), q\left(T_{m}\right)=\frac{(9 i(i+1))}{2}$, $i \in N$.

Remark 4.2. In a triangular snake $T_{m}, q\left(T_{m}\right)=3 m$ and $p\left(T_{m}\right)=2 m+1$, where $m$ is the number of blocks. By Theorem (4.1), $T_{m}$ admits CDPD if $q\left(T_{m}\right)$ $=\frac{9 i(i+1)}{2}, i \in N$. Therefore, $3 m=\frac{9 i(i+1)}{2}$ That is, $m=\frac{3 i(i+1)}{2}, i \in N . p\left(T_{m}\right)=$ $2 m+1=2\left[\frac{3 i(i+1)}{2}\right]+1$. That is, $p\left(T_{m}\right)=3 i^{2}+3 i+1, i \in N$.

Theorem 4.3. If $T_{m}$ admits $C D P D\left(P_{3}, P_{4}, P_{5}, \ldots, P_{3 i+2}\right)$, then $\gamma_{c}\left(T_{m}\right)$ $=\frac{3 i^{2}+3 i-2}{2}, i \in N$.

Proof. Let $D \subseteq p\left(T_{m}\right)$ is a connected dominating set in $T_{m}$. By our as- 
sumption, $T_{m}$ admits CDPD. let $v_{1}, v_{2}, v_{3}, \ldots, v_{\frac{3 i(i+1)}{2}}, v_{\frac{3 i(i+1)}{2}+1}$ be the base vertices of $T_{m}$. Then the cut vertices are $v_{2}, v_{3}, v_{4}, \ldots, v_{\frac{3 i(i+1)}{2}-1}, v_{\frac{3 i(i+1)}{2}}$. let $S$ $=\left\{v_{2}, v_{3}, v_{4}, \ldots, v_{\frac{3 i(i+1)}{2}-1}, v_{3 i(i+1)}\right\}$. Then $S$ is a minimum connected dominating set of $T_{m}$. Therefore, $|S|=|D|=\frac{3 i(i+1)}{2}-1=\frac{3 i^{2}+3 i-2}{2}$. That is, $\gamma_{c}\left(T_{m}\right)=$ $\frac{3 i^{2}+3 i-2}{2}, i \in N$.

Corollary 4.4. If $T_{m}$ admits $C D P D\left(P_{3}, P_{4}, P_{5}, \ldots, P_{3 i+2}\right), i \in N$, then $\gamma_{c}\left(T_{m}\right)=q\left(T_{m}\right)-p\left(T_{m}\right)$.

Proof. We know that $p\left(T_{m}\right)=3 i^{2}+3 i+1$ and $q\left(T_{m}\right)=\frac{9 i(i+1)}{2}, i \in N$. By Theorem(4.3), $\gamma_{c}\left(T_{m}\right)=\frac{3 i^{2}+3 i-2}{2}=\frac{9 i(i+1)}{2}-\left(3 i^{2}+3 i+1\right)=q\left(T_{m}\right)-p\left(T_{m}\right)$.

Theorem 4.5. If $T_{m}$ admits $C D P D\left(P_{3}, P_{4}, P_{5}, \ldots, P_{3 i+2}\right), i \in N$ and $\gamma\left(T_{m}\right)=|D|$, then

$$
\gamma_{c}\left(T_{m}\right)= \begin{cases}2 \gamma\left(T_{m}\right)-2 & \text { if } m \text { is odd } \\ 2 \gamma\left(T_{m}\right)-1 & \text { if } m \text { is even }\end{cases}
$$

Proof. Given that $\gamma\left(T_{m}\right)=|D|$. Therefore, $D$ is a minimum dominating set of $T_{m}$.

Case 1: $m$ is odd. $D$ consists of $\frac{1}{2}\left[\frac{3 i(i+1)}{2}+1\right]$ vertices. Therefore, $\gamma\left(T_{m}\right)$ $=\frac{3 i(i+1)+2}{4}$. Let $U$ and $W$ be two vertex sets such that

$$
U=\left\{u_{1}, u_{2}, u_{3}, \ldots, u_{m-1}, u_{m}\right\}
$$

and

$$
W=\left\{v_{1}, v_{2}, v_{3}, \ldots, v_{m}, v_{m+1}\right\} .
$$

Now, $N(D)=(W-D) \cup U$. Then $N(D)-U$ is a minimum dominating set of $T_{m}$. Take $F=N(D)-U$. Therefore, $(D \cup F)$ is a set of base vertices of $T_{m}$ and $|D \cup F|=\frac{3 i(i+1)+2}{2}=2 \gamma\left(T_{m}\right)$. Let $T=D \cup F$. Neglect the first and last vertices in $T$. Therefore, $T=(D \cup F)-\left\{v_{1}, v_{m+1}\right\}$ forms a minimum connected dominating set of $T_{m}$ and $|T|=2 \gamma\left(T_{m}\right)-2$. That is, $\gamma_{c}\left(T_{m}\right)=2 \gamma\left(T_{m}\right)-2$.

Case 2: $m$ is even. $D$ consists of $\frac{1}{2}\left[\frac{3 i(i+1)}{2}\right]$ vertices. Therefore, $\gamma\left(T_{m}\right)=$ $\frac{3 i(i+1)}{4}$. Let $X$ and $Y$ be two vertex sets such that $X=\left\{u_{1}, u_{2}, u_{3}, \ldots, u_{m-1}, u_{m}\right\}$ and $Y=\left\{v_{1}, v_{2}, v_{3}, \ldots, v_{m}, v_{m+1}\right\}$.

Now, $N(D)=(Y-D) \cup X$. Then $N(D)-X$ is a dominating set of $T_{m}$. Take $F=N(D)-X$. Therefore, $(D \cup F)$ is a set of base vertices of $T_{m}$ and $|D \cup F|=\frac{3 i(i+1)}{2}+1=2 \gamma\left(T_{m}\right)+1$. Let $T=D \cup F$. Neglect the first and last 
vertices in $T$. Therefore, $T=(D \cup F)-\left\{v_{1}, v_{m+1}\right\}$ forms a minimum connected dominating set of $T_{m}$ and $|T|=\left[2 \gamma\left(T_{m}\right)+1\right]-2=2 \gamma\left(T_{m}\right)-1$. That is, $\gamma_{c}\left(T_{m}\right)$ $=2 \gamma\left(T_{m}\right)-1$.

Illustration 4.6. The following table illustrate (see Table 1) the CDPD, connected domination number $\left(\gamma_{c}\right)$ and domination number $(\gamma)$ of $T_{m}$.

\begin{tabular}{|c|c|c|c|c|c|}
\hline$m$ & $p$ & $q$ & $\mathrm{CDPD}$ & $\gamma_{c}$ & $\gamma$ \\
\hline 3 & 7 & 9 & $P_{3}, P_{4}, P_{5}$ & 2 & 2 \\
9 & 19 & 27 & $P_{3}, P_{4}, \ldots, P_{8}$ & 8 & 5 \\
18 & 37 & 54 & $P_{3}, P_{4}, \ldots, P_{11}$ & 17 & 9 \\
30 & 61 & 90 & $P_{3}, P_{4}, \ldots, P_{14}$ & 29 & 15 \\
45 & 91 & 135 & $P_{3}, P_{4}, \ldots, P_{17}$ & 44 & 23 \\
63 & 127 & 189 & $P_{3}, P_{4}, \ldots, P_{20}$ & 62 & 32 \\
84 & 169 & 252 & $P_{3}, P_{4}, \ldots, P_{23}$ & 83 & 42 \\
108 & 217 & 324 & $P_{3}, P_{4}, \ldots, P_{26}$ & 107 & 54 \\
135 & 271 & 405 & $P_{3}, P_{4}, \ldots, P_{29}$ & 134 & 68 \\
165 & 331 & 495 & $P_{3}, P_{4}, \ldots, P_{32}$ & 164 & 83 \\
198 & 397 & 594 & $P_{3}, P_{4}, \ldots, P_{35}$ & 197 & 99 \\
234 & 469 & 702 & $P_{3}, P_{4}, \ldots, P_{38}$ & 233 & 117 \\
273 & 547 & 819 & $P_{3}, P_{4}, \ldots, P_{41}$ & 272 & 137 \\
315 & 631 & 945 & $P_{3}, P_{4}, \ldots, P_{44}$ & 314 & 158 \\
360 & 721 & 1080 & $P_{3}, P_{4}, \ldots, P_{47}$ & 359 & 180 \\
\hline
\end{tabular}

Table 1

Theorem 4.7. If $T_{m}$ admits $C D P D\left(P_{3}, P_{4}, P_{5}, \ldots, P_{3 i+2}\right)$, then

$$
\gamma_{c}\left(T_{m}\right)=\sum_{j=1}^{3 i} \gamma_{c}\left(G_{j}\right)-\left(3 i^{2}+1\right), \quad i \in N
$$

Proof. By our assumption, $\gamma_{c}\left(G_{1}\right)=1, \gamma_{c}\left(G_{2}\right)=2, \ldots, \gamma_{c}\left(G_{3 i}\right)=3 i, i \in N$. 
Then

$$
\begin{aligned}
\sum_{j=1}^{3 i} \gamma_{c}\left(G_{j}\right)-\left(3 i^{2}+1\right) & =\left[\gamma_{c}\left(G_{1}\right)+\gamma_{c}\left(G_{2}\right)+\ldots+\gamma_{c}\left(G_{3 i}\right)\right]-\left(3 i^{2}+1\right) \\
& =[1+2+\ldots+3 i]-\left(3 i^{2}+1\right) \\
& =\frac{3 i(3 i+1)}{2}-\left(3 i^{2}+1\right) \\
& =\frac{3 i^{2}+3 i-2}{2}
\end{aligned}
$$

Then

$$
\sum_{j=1}^{3 i} \gamma_{c}\left(G_{j}\right)-\left(3 i^{2}+1\right)=\gamma_{c}\left(T_{m}\right)
$$

by Theorem 4.3 .

\section{References}

[1] Y. Alavi, G. Boals, G. Chartrand, P. Erdos, and O.R. Oellermann, The ascending subgraph decomposition problem, Cong. Number., 58 (1987), 7-14.

[2] Dushyant Tanna, Harmonius labelling of certain graphs, In. J. Adv. Engg. Res. Studies, II/IV (2013), 46-48.

[3] N. Gnanadhas and Paul Raj Joseph J., Continuous monotonic decomposition of graphs, International Journal of Management and System, 16, No. 3 (2000), 333-344.

[4] F. Harary, Graph Theory, Narosa Publishing House, New Delhi, 1998.

[5] Juraj Bosak, Decomposition of Graphs, Kluwer Academic Publishers, 1990.

[6] K. Lakshmi Prabha, K. Nagarajan, Ascending domination decomposition of graphs, International Journal of Mathematics and Soft Computing, 4, No. 1 (2014), 119-128.

[7] M.H. Muddebihal, Majeed Abdul, Connected domination in block subdivision graphs of graphs, Mathematical Theory and Modelling, 2, No. 6 (2012).

[8] E. Sampathkumar, H.B. Walikar, The connected domination number of a graph, Jour. Math. Phy. Sci., 13, No. 6 (1979), 607-613. 
